\title{
Providing Process Origin Information to Aid in Computer Forensic Investigations*
}

\author{
Florian P. Buchholz \\ CERIAS \\ Purdue University \\ florian@cerias.purdue.edu
}

\author{
Clay Shields \\ Department of Computer Science \\ Georgetown University \\ clay@cs.georgetown.edu
}

\begin{abstract}
The number of computer attacks has been growing dramatically as the Internet has grown. Attackers currently have little or no disincentive to conducting attacks because they are able to hide their location effectively by creating a chain of connections through a series of hosts. This method is effective because most current host audit systems do not maintain enough information to allow association of incoming and outgoing network connections. In this paper, we introduce an inexpensive method that allows both on-line and forensic matching of incoming and outgoing network traffic. Our method makes small modifications to the operating system that associate origin information with each process in the system process table, and enhances the audit information by logging the origin and destination of network sockets. We present implementation results, show that our method can effectively record origin information about a variety of attacks, and describe the limitations of our approach.
\end{abstract}

\section{Introduction}

As the Internet has become a widely accepted part of the communications infrastructure there has been an increase in the number of network attacks [7]. One factor in the growth of attacks is that network attackers are only rarely caught and held accountable for their actions, giving them relative impunity in action. This situation has arisen, in part, because of the relative ease that attackers have in hiding their location, making it difficult and expensive for investigators to determine the origin of an attack. The easy availability of sophisticated tools, coupled with this lack of accountability, has lead to a situation in which Internet attackers can and do repeatedly strike with impunity.

To draw an analogy, this is like a neighborhood responding to a series of muggings by simply having the victims and other concerned citizens buy armor

${ }^{*}$ Published in Journal of Computer Security, Vol. 12(5), pp. 753-776, September 2004 
or take self-defense classes. While this might improve individual resistance to attack, members of the community who were unwilling or unable to acquire such measures, or who were simply slow to do so, would be still be vulnerable to the free-roaming muggers. Because the thieves are not held accountable for their actions, there are no disincentives for them not to strike again. A better solution is for the community to take action to find the attacker and hold them accountable for their actions so that the attacks decrease or stop. The work presented here is a step in that direction.

In this paper we present an extended discussion of a simple and inexpensive method for maintaining the necessary information to correlate data entering a host with data leaving a host that first appeared at the 2002 Usenix Technical Conference [4]. The goal of this work is to provide additional audit data that can help determine the source of network attacks. We include results from an implementation for the FreeBSD 4.1 kernel that show the technique is effective in providing information useful in tracing common attack situations, particularly for tracing stepping stones and denial-of-service attack zombies.

\section{$2 \quad$ Background}

The goal of network traceback research is to allow attack attribution, so that the source of attack traffic, which is a particular host used by a human to initiate an attack, can be identified and appropriate real-world investigative techniques used to locate the person responsible.

In general, attackers use two different methods to hide their location [17]. One method, common in denial-of-service attacks, is to spoof the source address in IP packet headers so that recipients cannot easily determine the true source. As discussed further in Section 7, this has been an area of significant research in recent years, and a number of proposals for tracking and filtering such traffic have been presented.

The other method, which has received significantly less attention from the research community, is for attackers to sequentially log into a number of (typically compromised) hosts, as shown in Figure 1. These forwarding hosts, often called stepping-stone hosts [44], effectively disguise the origin of the connection, as each host on the path sees only the previous host on the connection chain. This is an effective method of providing anonymity, similar to the mechanism used in cooperative protocols designed to provide privacy through anonymity $[29,28,18]$. A victim of an attack therefore might not be able to determine the original source of an attack without tracing the path back through all intermediate stepping-stone hosts.

There have been two general mechanisms proposed to do this. The first mechanism, named stream matching, attempts to follow the path by examining the flow of data through the network. As described further in Section 7, this seems to be a promising technique, though no definitive method or rate of accuracy has been determined. These techniques also seem vulnerable to the attacker manipulating characteristics of the data flow. 


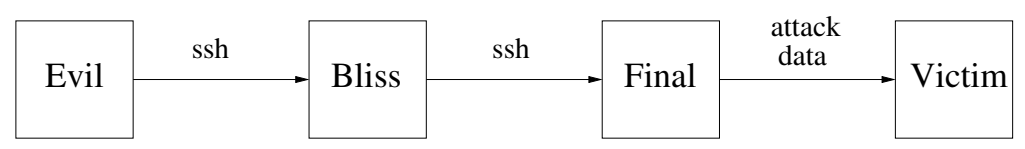

Figure 1: A sample connection chain

The more common method of tracing an attack is to sequentially visit each host on the connection chain, and to examine the state of the machine or its logs for evidence about the prior hop. This can be difficult. First, attackers who compromise systems take steps to hide their activity. This includes modifying the operating system to not report their running processes, and altering or deleting $\log$ entries to hide the address of the machine they connected from. Protecting the operating systems and its logs is a common problem and is generally outside the scope of our work. We assume that the system logs are protected by cryptographic mechanisms [32] or by the more common techniques of being written to write-only media or being sent to a more secure centrallogging facility. In the event of the system or logs being compromised, however, tools like The Coroner's Toolkit [12] can be used to attempt to discover hidden and deleted files and use access times to deduce system activities. Such forensic analysis may not recover adequate information for traceback if it did not exist in the first place, or was overwritten by the attack or the operating system.

The larger problem, and the one we address with our work, is that systems generally do not log the information necessary to trace a connection back to its source, so that even if logs are protected or recovered, there may be inadequate information to trace the connection back. While most systems track users by the times they were logged in and from where, they do not track what outgoing connections a user initiates. This means that while it may be easy to track an intruder through a system that has only one or a few users, it can become very difficult to track an intruder across a system that has many users. This is because even though an investigator can determine which users were logged on, it may be impossible to determine which individual is responsible for the outgoing network traffic that created the next step. The investigator might then need to examine many different possible last hops corresponding to all users on the system at the time - a task that can be daunting or impossible. The goal of our work is to create mechanisms to address this problem, and allow forensic association of outgoing traffic with the source of incoming traffic which created it.

\section{Host causality}

Though certain aspects of the network traceback problem have been addressed, a new area of research that is concerned with data transformations or data flow tracking through a host is needed for a complete picture for attack origin traceback. We call this new area host causality, because we are attempting to 
determine what network input causes other network output across a host.

Common operating systems do not currently provide information that can match incoming and outgoing network traffic. While there has been some work that attempts to use existing system information to match active incoming and outgoing streams $[16,6]$, this work has been either shown to be impractical to securely implement [3], or requires an external trigger to store forensic information. Ideally, it should be possible to determine whether network traffic was originated directly from a particular host or occurred as a result of a connection from some other remote machine, and, if possible, which remote machine is involved. This would not only help in tracing back to the source of a network attack, but could be useful in showing due diligence, so that the owner of a machine used in an attack could demonstrate that the attack originated elsewhere.

A solution that addresses the problem of tracing connections through a host is necessary because a host on the network can transform data passing through it in such a way that, from the network's point of view, it can no longer be easily related to traffic leaving it. This might be the case in a stepping stone scenario, if the traffic is delayed, or differently compressed or encrypted. Also, in attacks like a distributed denial-of-service (DDoS) attack [43], control traffic cannot be linked to the resulting attack traffic. In such an attack, packet source-location techniques might identify the source of a particular attack stream, but will not allow identification of the master or the controlling host. This is due to the fact that the datagrams that are used to perform the attack are seemingly unrelated to those that control the client. What is missing is information within the host that can be used to associate an incoming control packet with outgoing attack data.

\subsection{Required and Desired Properties}

The following properties need to be fulfilled in order to achieve a practical solution to the host causality problem:

- It must be possible to determine whether a given process on the host was started by a local user or remotely.

- If a process was started by a user at a remote location, information about that source must be maintained and associated with the process.

- An audit facility must exist that allows the logging of incoming network traffic and processes that receive it. This will allow correlation between the source of a process and the source of incoming network packets.

- An audit facility must exist that allows the logging of individual outgoing network traffic and processes that send it. Combined with the facility above, one could then relate incoming and outgoing traffic processed by the same process. 
- Processes that spawn other processes need to pass on their source information to their children, or, if they provide a remote login service, pass on the remote location as the child's new source.

Furthermore, the following properties are desirable so that a correct and efficient correlation is possible on a system:

- The logs maintained about origin information should be resistant to modifications by attackers. This is a general problem with many types of logs, and other work addresses this problem [32].

- The modifications to a system should be minimal so that they do not interfere with existing software.

- Due to restricted logging space, it should be possible to use rules to control what data the audit system collects.

- It should be possible to quickly identify processes that were not started locally together with their remote location.

\section{Host Causality Mechanisms}

A process on a computing host is an executing instance of a program [37]. Processes are therefore, among many other things, responsible for receiving and generating network data on a host that is connected to a network.

Processes can be started:

- explicitly by a human being

- by the system

A human being can start processes:

- while physically present at the host

- from a remote location

- indirectly through some other process he or she started

The system can start processes:

- through startup scripts (including init and .profile)

- through scheduling services like cron and at

- through system services like inetd or sshd

The origin of a process is the information about how any process running on the system was started in regard to the above possibilities. For the purpose of this paper only a distinction between a process that was started by a human being from a remote location (remote origin) and the other ways (local origin) is of importance, with the exception of the special case of indirectly started processes. 
In case of a remote origin for a process, the origin information should include that remote location. If the system tracks the origin of a process and it is of remote origin and sends out network traffic, then the system can make a connection between the traffic that was sent out and the traffic that was received from the origin of the process over the network. The traffic could be individual datagrams, or they can be part of an established connection.

In order to gain access to a system from a remote location and start new processes there, a user has to make use of a service offered on that particular system. Usually most systems provide well-known services such as telnet, rsh, or ssh that will give a remote user a shell on the system. However, there are other possibilities to create new processes that do not involve an interactive shell. In fact, any process listening on an open port on the system may be used or misused for such purposes. Our solution does not address these problems, and they are a topic for future investigation.

As the only legitimate remote access to a system is through its well-known services, it is feasible to store information about the existing connection with the newly created child process. After a successful login procedure, the source of the new process should reflect the information stored about the connection. Note that the origin of a process and its subsequent children is set at the time a user gains access to the system. All programs that will be started during that remote session will inherit that origin. At this point time delays become irrelevant, as origin information is stored with the processes no matter whether or not processes become dormant for any amount of time.

\subsection{Information Storage}

From the viewpoint of a host, all that can be deduced about the origin of an arriving network packet is the interface that it arrived on and the information that is contained in the packet itself. A host on its own cannot determine whether a network datagram was spoofed or not. Therefore, for IP packets, the five-tuple consisting of source and destination IP addresses and source and destination ports and the protocol number must suffice to distinguish source information maintained about processes on a host. If packet traceback schemes are deployed and can provide additional information, it is possible to maintain that information as well.

While storing information about active processes can be useful, for complete analysis of attacks, some additional information needs to be logged as well. The logging mechanism can maintain more explicit information than simply storing the IP five-tuples. Along with the five-tuple and timestamp, the system can also store the interface on which the packet arrives and the process id. If the system logs individual packets, it can also store a checksum of the non-changing parts of each packet header and maybe even the payload of each packet that is logged in case the need for a more detailed post-analysis matching arises. Answers to questions like: "Have you seen this packet?" could then be easily answered. Furthermore, it would be expensive and impractical to log an entire stream of packets that make up the entirety of a TCP stream. Since TCP is a connection 
oriented transport layer protocol, it is sufficient to only regard incoming and outgoing SYN requests for the purposes of logging.

Unfortunately, UDP is a connection-less protocol. Thus for UDP, all packets might need to be logged. Depending on the level of detail that is desired, however, one can apply certain log-reducing mechanisms. For example, one could exclude UDP packets to and from well-known services that heavily utilize UDP, such as NFS or syslog. To reduce logging for large amounts of UDP packets, such as the traffic that a DoS client might generate, one could log only the first packet of a UDP stream and keep a count of packets sent as part of that stream in the future. Each new packet of the stream would extend a time interval which, once expired, would trigger the log entry to be written with the accumulated information.

\subsection{Limitations on Information Availability}

For well-known services we can assume that there will only be one open network connection for each child process spawned. Non-standard server programs might behave differently, however, and there might be multiple open connections when we try to determine the origin information. In this case, it is impossible to be sure which connection should be considered as the origin of the process. Because of this, there can be a problem with using the latest data from the accept system call as the origin information. If a server program allows multiple open sockets before the call to login, then there is a possibility that the wrong origin information is stored with the process. It is possible to design a program that after accepting a connection opens another listening socket to receive a decoy connection from a completely different remote site or, even worse, from the local host itself. This would set the information obtained from the accept call to the new socket's source data, before login was invoked. After a successful login procedure, the origin information would be incorrect. If a local user installs such a program, then any attacks originating from it can be viewed as originating from the host, which is consistent with our definition of local origin.

Another problem is that a remote user may still hide his real origin by creating a connection from the system to itself. In this case the origin information of the process gets changed to the source information of the local host. While the process is still being considered of remote origin, it is of no value from a traceback perspective. If many remote processes "change" their origin in such a fashion, one cannot determine anymore what the "real" origin of any of those was. In order to prevent this obscuring of the origin of a process, one needs to $\log$ an inheritance line for remote processes. Starting from a process with remote origin, each time the process or one of its children spawns another child process, this information needs to be recorded, with an entry such as "Process $x$ spawned process $y$ ". Instead of logging only the process numbers of parents and children when processes fork, a simple extension would be to add the origin of the parent process to the log entry. This would establish a chain of ancestors for all processes that originated from a process of remote origin, and this chain could easily be reconstructed from the logs. Using this technique, even if any 
of the child processes attempt to change their origin to a local connection the original origin also can be reconstructed from the logs.

\section{Implementation}

The model described above was implemented in the FreeBSD 4.1 operating system on a PC with an $\mathrm{x} 86$ architecture. While the implementation is therefore specific to the UNIX operating system, the general principles of the model should be applicable to other systems as well.

All processes that accept network connections do need to make use of the socket system calls provided by the system. Stevens [36] describes the necessary steps to set up a TCP or UDP server. They involve system calls to bind, listen, and accept (recvfrom in the case of UDP), in that order. Thus any connection between two systems must have successfully undergone a call to accept on the server side. In the case of TCP, accept returns after a successful three-way handshake. In the case of UDP, recvfrom returns upon reception of a packet that matches the socket characteristics.

As a successful connection implies a successful return from the accept system call, it seems reasonable to make modifications there in order to obtain location information. Specifically, with the assumption of only one open network connection, it is sufficient to record the data from the last call to accept. This information will then be accessible to the child process created by the fork system call. Finally, after a successful login procedure, the source of the new process should reflect the information stored about the connection. As the login program lies in user space and not all well-known servers utilize it, it will be necessary to perform this step through one of the system calls such as setlogin. All user-space programs and also all libraries that provide login services (i. e., changes of real and effective user IDs) use those system calls, so it is sufficient to modify the system calls.

All the necessary information described in Section 4 is available within data structures used by the accept system call. Once the connection has been established, the socket descriptor contains the source IP address and the source port of the purported source of the traffic. To determine the destination IP address and port that was used to establish the connection, the system also has to access the protocol control block $(\mathrm{PCB})$ that is associated with the socket and that is pointed to from the socket data structure ${ }^{1}$. The information can be obtained through simple pointer lookups.

\subsection{Where to store source information}

We decided to maintain the information directly in the process table itself, because it is simple to add another field that contains the necessary information, and creation and termination of processes is handled automatically. The inheritance problem is taken care of as well, as the fork system call causes certain

\footnotetext{
${ }^{1}$ See McKusick et al. [19] or Wright and Stevens [39] for further details.
} 
fields of the process table to be copied to the child. The only time we therefore need to access the field in the process table is when origin information changes. The disadvantage of this approach is that some auxiliary programs such as top and ps might have to be adjusted to accommodate the changes.

It is possible to utilize existing logging facilities, such as syslog to record the data, or a logging program can develop its own format and location to store the information [26]. Ideally, there would be some mechanism to ensure the integrity of the logs. Write-once, read-many media, or a secure logging facility could be used [32].

\subsection{Data structures and kernel modifications}

For the source information, a new data type, struct porigin, was created as shown in Figure 2. The type field denotes whether the source is local (0) or remote (1). If the type is 0 , all other fields are undefined and can be ignored. The next five fields are the typical four-tuple for a TCP or UDP connection, consisting of source and destination IP addresses as well as source and destination ports, plus the protocol number. The last parameter is a timestamp, which denotes the time the connection was established in network time format [20].

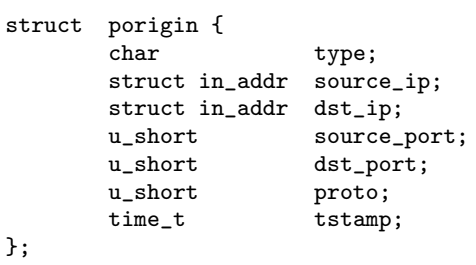

Figure 2: The process origin data structure

Note that the network interface is not included here. In most cases it can be obtained with the information stored if necessary. In some situations, though, this is not possible. For these cases, it is desirable to have the interface information available as part of the origin information. However, this would require non-trivial modifications of the network stack data structures, as that information is not retained as data is passed among the stack levels. A special implementation for hosts where the interface information cannot be determined by the 4-tuple might be the best solution.

In order to keep track of the corresponding source information for each process, the process table data structure (struct proc) was modified in two locations, as shown in Figure 3. It is necessary to retain the actual source information as well as information about the last accepted connection of a process. The latter is needed because all common TCP/IP based network services that provide a remote login facility first accept the connection and then fork off a child process where login is called. Hence, two fields, origin and lastaccept were added to the process table structure, both of type struct porigin. The 
fields are located in the area that gets copied in the fork system call.

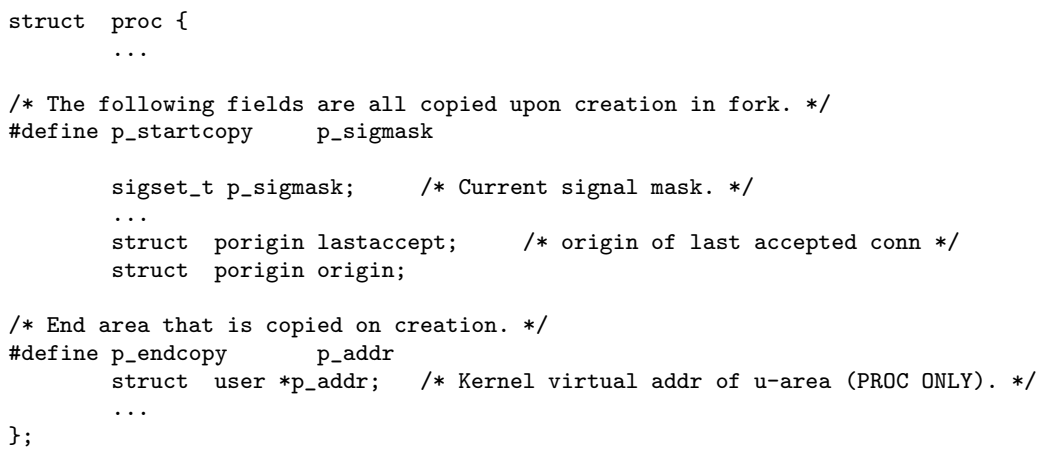

Figure 3: The modified process table data structure

The copying of the origin field provides a simple and elegant solution for the inheritance mechanism. All it takes is a few more bytes to be copied in the fork system call, as the process structure is copied anyway. Thus, a child process always inherits the source information from its parent.

This leaves the question of where the two fields, lastaccept and origin are to be set. As the name already suggests, lastaccept is set in the accept system call, after a successful accept of an incoming connection. The modified accept system call was implemented as shown in Figure 4, which shows how to retrieve information from the $\mathrm{PCB}$.

Note that accept 1 is called from the actual accept system call. The connection will be accepted in the procedure soaccept. If the call is successful, the type is set to 1 , and the four-tuple is obtained from the PCB associated with the socket via the pointer inp. Note that this will only work for a TCP connection, which is used by services which provide a shell. For future work, other protocol types need to be considered. For instance, in the case of UDP, the recvfrom system call may be modified in a similar fashion. Since there are no well-known services that provide a shell and utilize UDP, however, the modification of TCP's accept is sufficient for our purposes. Section 8 discusses the future work in more detail.

The origin field will be copied from a parent process to its child. However, as discussed above, each time a login is performed within a process, the source information of the last accepted socket should become the new origin information for that process. Thus, at an invocation of login, the lastaccept field should be copied into the origin field. However, as discussed above, login is only a program in user space that simply utilizes several system calls to perform the actual user login. One could supply a separate system call to have the lastaccept field copied to the origin field, but that would imply that every program that supplies a login service to be changed and use it. Therefore, one of the system calls used by every login service, setlogin was modified so that the field is copied after a successful call. 


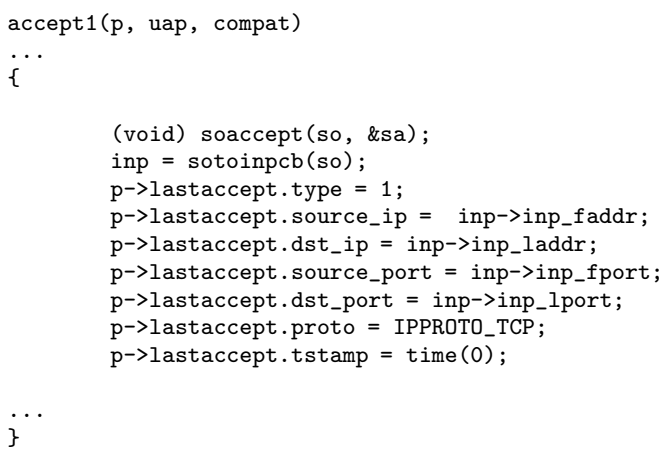

Figure 4: The modified accept system call

To keep track of the inheritance line for a remote process, it is necessary to modify the fork system call, as well. It is sufficient to record the process IDs of the parent and child processes in case the parent is of remote origin. From this information, it is possible to reconstruct the entire inheritance line for a remote process up to the first parent that was of remote origin. The syslog facility provides an easy way to log kernel messages, and was chosen to record the information out of reasons of simplicity. Figure 5 shows the modifications made to fork. In future work, this recording mechanism needs to be refined and optimized.

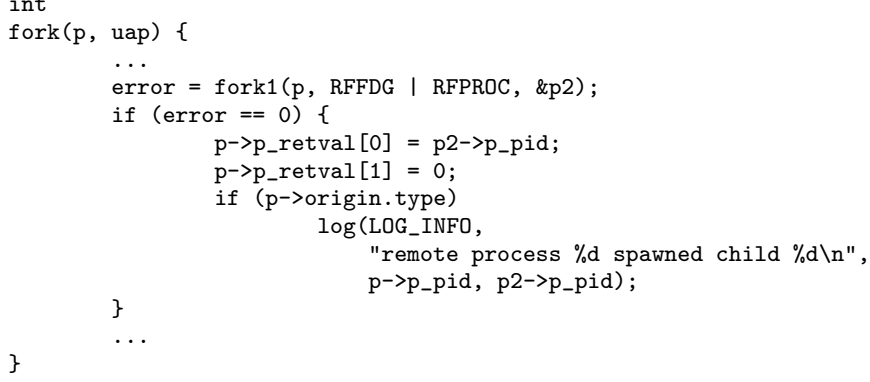

Figure 5: The modified fork system call

\subsection{System calls}

In order to access the source information for a given process, a new system call, getorigin was added to the system. It takes as parameters a process identifier and a buffer, into which the source information is copied. The getorigin call is shown in Figure 6. Note that there is no system call to set or reset the origin field. With the getorigin system call, it is now possible to design 
logging facilities and administrative programs within user space that make use of the source information of a process. For reasons of simplicity, the call was implemented to be unrestricted.

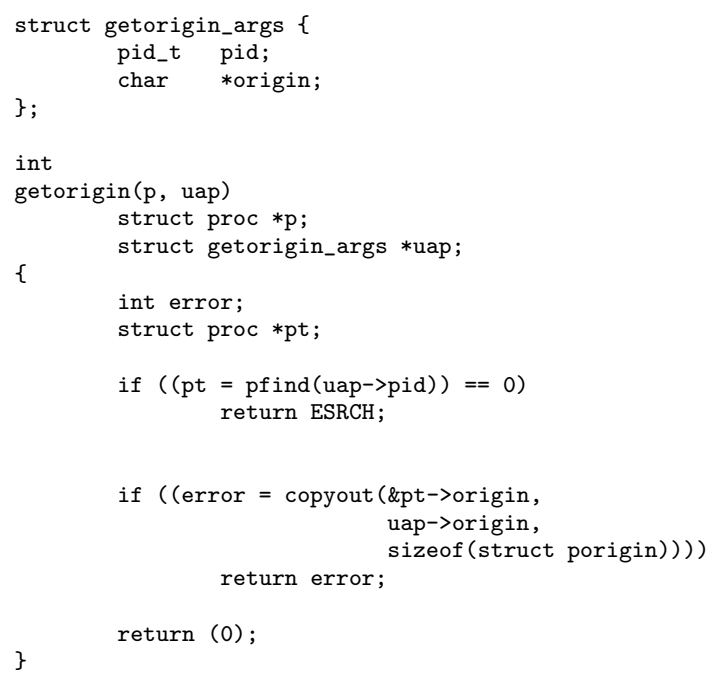

Figure 6: The new getorigin system call

Another system call, portpid, was added to give support for the logging facility described below and is shown in Figure 7. If one wants to associate incoming TCP or UDP packets with the receiving process, one needs to find the process id of the socket that will handle an IP packet. The same is true for sockets that are responsible for outgoing packets. Those sockets are identified in the network layer by the four-tuple of source and destination addresses and ports, but, unfortunately, there is no efficient mechanism in FreeBSD to obtain that information within user space. It is possible to determine the open socket for a given process id but there is no direct lookup from socket to process id. Thus, in order to avoid iterating through all processes on a system, the system call portpid will take a four-tuple as well as a protocol identifier (TCP or UDP) and will return the process id of the process that belongs to the listening socket that will accept packets matching the four-tuple, or belonging to the socket that sent the packet. If there is no such socket, an error will be returned. A weakness of this design is that a process may exit and be removed from the process table before the portpid call occurs. This problem is detailed in Section 8 below.

The FreeBSD operating system uses protocol control blocks (PCBs) to demultiplex incoming IP packets. The PCBs are chained together in a linked list and contain IP source and destination addresses and TCP or UDP ports or wildcard entries for incoming packets to match against. Each PCB also contains a pointer to the socket that is destined to receive a packet, should it match the four-tuple specified in the PCB. From the socket, one can then look in the 


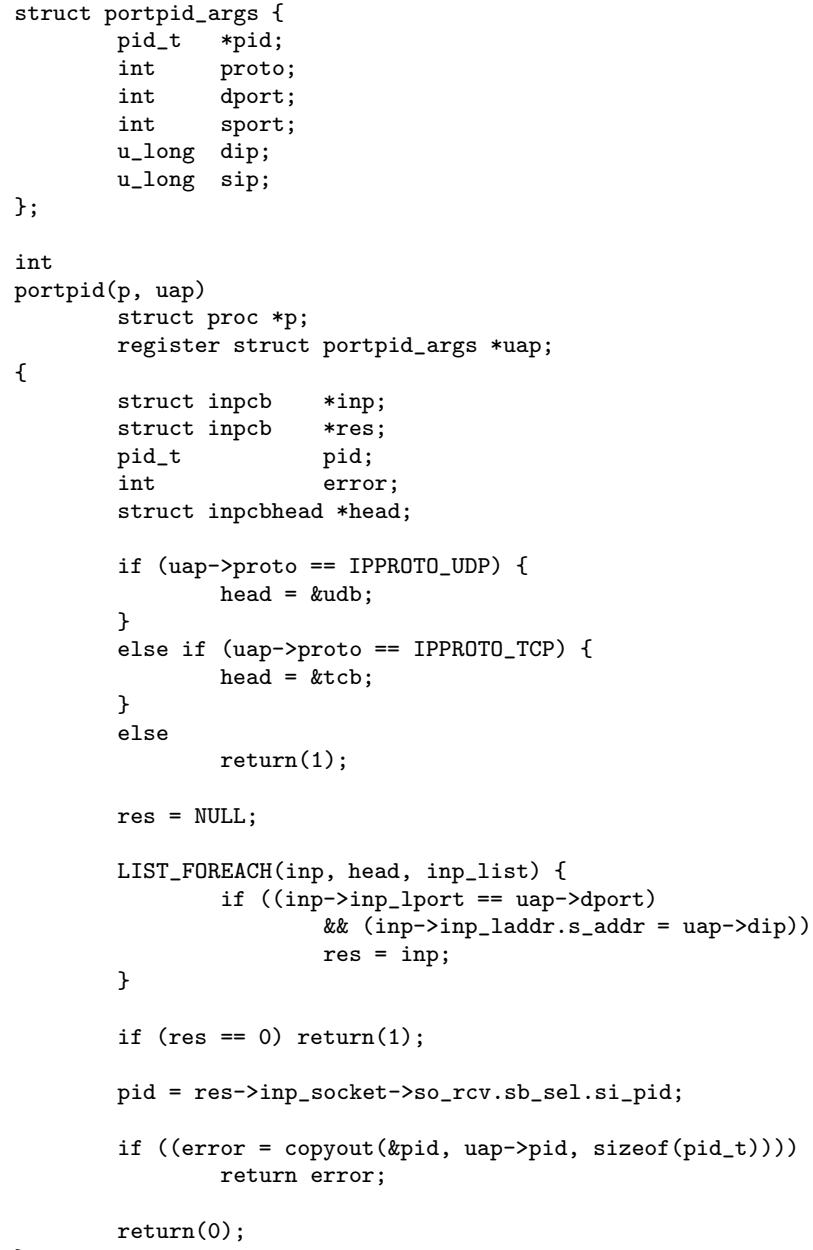

Figure 7: The new portpid system call 
receive or write buffer to obtain the actual process id of the receiving or sending process, respectively. In order to determine which process will receive a packet or which process sent a packet, one needs to traverse the list of PCBs until the best match is found, and then obtain the process id of the socket associated with the PCB.

\subsection{Logging facility}

The logging facility that was implemented is merely a proof of concept, and there are many feasible ways to design and implement one. Our implementation of the logging facility uses the libpcap library, which is part of the Berkeley Packet Filter (BPF). The BPF will make a copy of each incoming and outgoing network packet that matches given filter criteria and supply that copy to the process utilizing the filter.

This prototype logging facility can therefore be considered as a network sniffer, but a more robust and efficient implementation would be one that is part of the kernel itself. For each TCP SYN or UDP packet seen by the sniffer, the portpid system call is invoked to obtain the process id of the process responsible for the packet. Once the process id is obtained, getorigin is called for that process id to determine whether the process is of remote origin or not. If it is of remote origin, then the packet as well as the origin information is printed out. Figure 8 shows the interaction of the different parts of the system with the logging facility, and Figure 9 shows the important parts of the routine that processes the packets passed on by the BPF. For better readability, unimportant parts such as the conversion of IP addresses to strings have been omitted.

In our prototype, there is a problem with logging outgoing UDP packets. The portpid system call relies on the socket that sent the packet to be still open so that it can find it in the PCB list. If an application opened a socket, wrote one UDP packet, and immediately closed the socket again, there would be a chance that the socket no longer existed when the packet was examined by the logging facility. DDoS clients usually keep the socket they send packets from open so that packets can be sent at a faster rate, but for outgoing control packets, this is a limitation. For TCP, this is not a serious issue, as there is either a three-way handshake or a time-wait period at the end of each connection.

One method to solve the outgoing UDP packet problem could entail further modification of the kernel, keeping the process ids of sending processes in a cache and making that information available to the portpid system call. A similar approach could also improve lookup performance for incoming packets. Instead of duplicating the de-multiplexing effort made in the networking stack, modifications to the stack could result in a new data structure that returns the correct process id for a given five-tuple. 


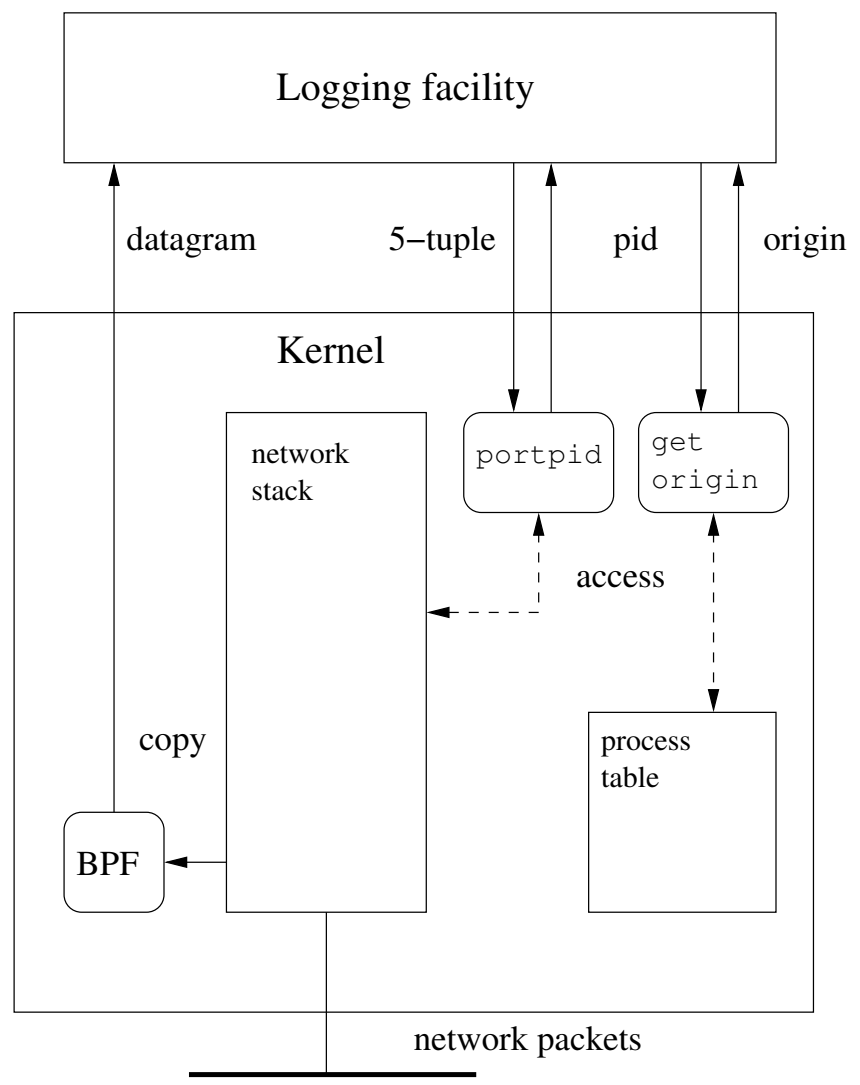

Figure 8: The logging facility 


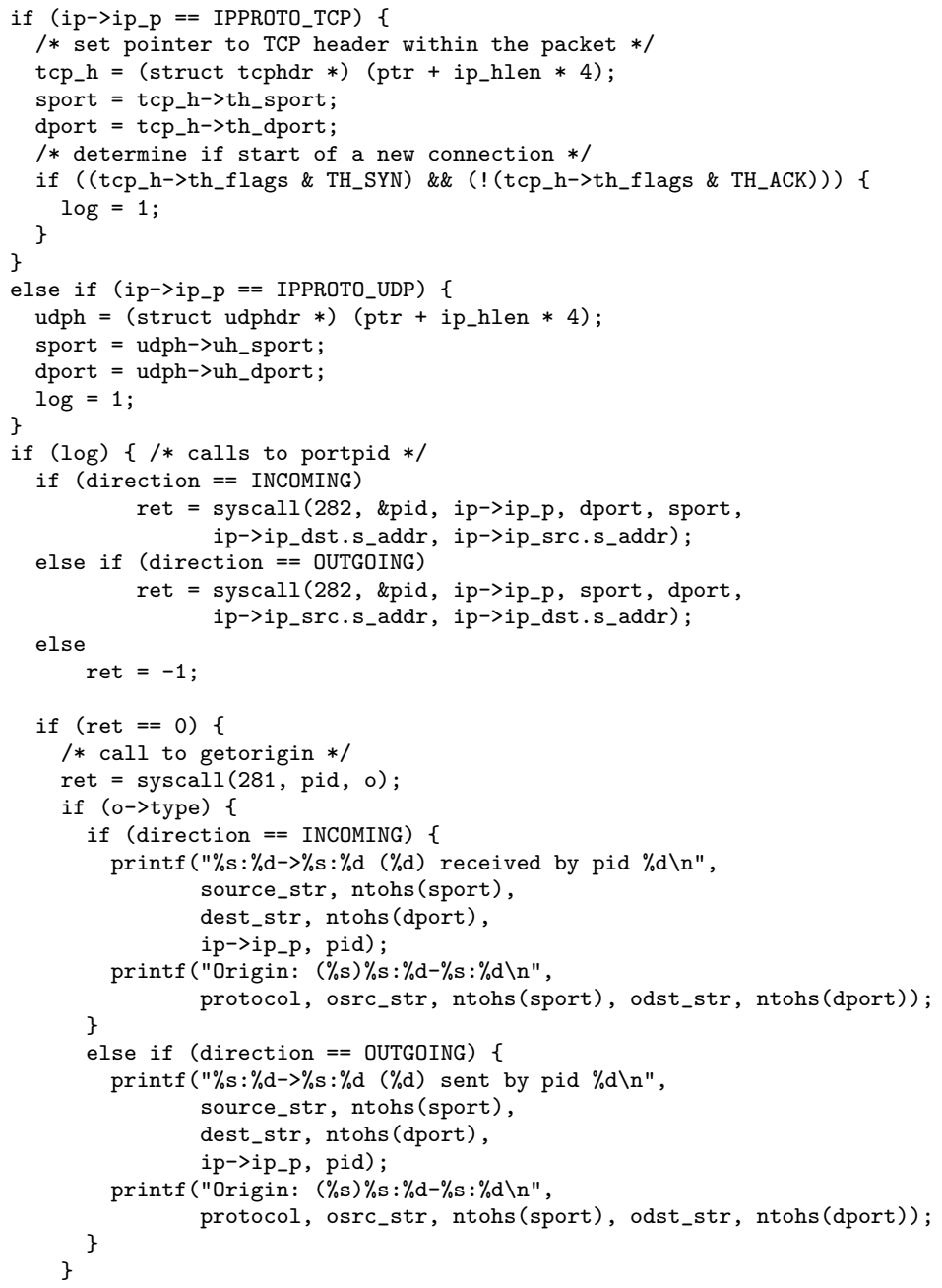

Figure 9: The packet processing routine of the logging facility 


\section{Implementation Results}

The modified kernel was installed on an Intel Pentium III Celeron PC. The machine used is part of a small networking lab. We will discuss the effects of the changes on the normal system behavior as well as give two examples of processes of remote origin handling traffic.

\subsection{Effects on normal system behavior}

As the changes to the system were only few and cheap, the impact on the system is minimal. The getorigin copies a few bytes from the process table and is only executed for TCP SYN and UDP packets. For those packets, the call to portpid causes a linked-list traversal of the protocol control blocks in the same manner the networking stack does its de-multiplexing. In every call to accept, the lastaccept field is set from the socket information. These operations are very few and inexpensive compared to the entire set of operations within accept. In every call to fork, an extra few bytes need to be copied to pass on the origin information to a process's child. The way the syslog facility was used to keep record of an inheritance line is very inefficient. On a system where processes spawn many children, the logs may quickly wrap around. That and the fact that the inheritance line needs to be reconstructed manually from the logs suggests the need for a re-design of the inheritance line for future work, as described in Section 8 .

\subsection{Examples}

\subsubsection{Stepping Stone}

In this example, bliss was used as a stepping stone. A user from evil (10.0.0.1) logged into bliss (192.168.0.1) via ssh. From there, he used ssh again, to log into final (172.16.0.1). The actual host names and IP addresses have been replaced by fictitious ones. This setup is equal to the example given in Figure 1 with the exception of the very last host.

The logging facility recorded the following entry from this:

$$
\begin{aligned}
& \text { 192.168.0.1:1022->172.16.0.1:22 (6) sent by pid } 285 \\
& \text { Origin: (TCP) } 10.0 .0 .1: 1022-192.168 .0 .1: 22
\end{aligned}
$$

One can observe, that the origin information indicates the connection from evil, port 1022, to bliss, port 22 (ssh). The logging mechanism didn't log the connection from evil to bliss, as sshd is a local process. However, evil is clearly shown as the origin for the process that connected to final. Therefore one can now associate the stream from bliss to final to the one from evil to bliss for traceback purposes.

\subsubsection{DDoS Client}

In this example, a distributed denial-of-service trinoo client was obtained from the Packet Storm archive [23] and was installed on bliss from evil as shown 
in Figure 10. The corresponding master was installed on another machine, master (192.168.0.2). Bliss was then used via master to perform a denial of service attack against victim (172.16.0.2), a third machine in the test network. Again, host names and IP addresses have been changed. A sample of the logging output is presented in Figure 11.

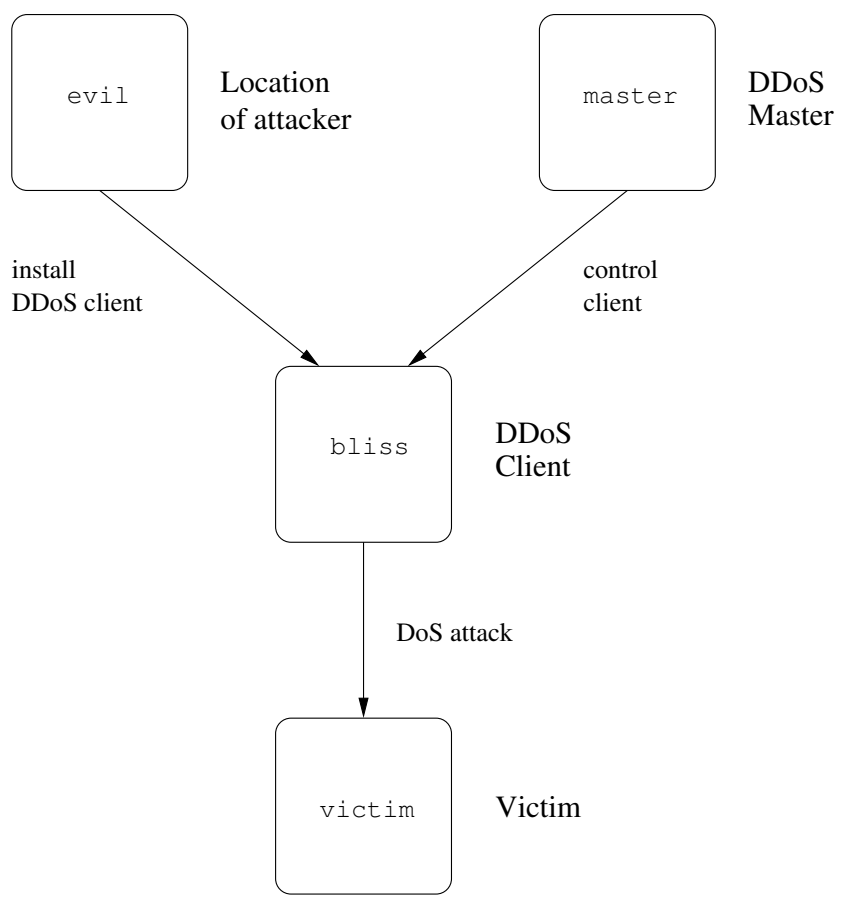

Figure 10: Setup for the DDoS attack

The first logged event is a UDP packet from bliss to master, notifying the trinoo master that a client is active. The next event is then a UDP packet from master to bliss, triggering the DoS attack. The rest of the log shows UDP packets sent from bliss to victim as part of the attack.

All the traffic can be unambiguously associated with the process 3760 , the DDoS client. From the origin, one can see that the process was started from evil. In this example, it is clear that the attack was controlled from master. This might not always be possible, as multiple packets from different locations could be received by the process just before an attack. However, by examining the logs a good estimate might be derived. At the very least it will give a list of possible hosts from where the attack was launched, which could be used to determine the location from where the software was set up and the master could now be investigated in the same manner as bliss to determine more information about the attack and the location of the attacker. 
192.168.0.1:1117->192.168.0.2:31335 (17) sent by pid 3760

Origin: (TCP) 10.0.0.1:32155-192.168.0.1:13419

192.168.0.2:39805->192.168.0.1:27444(17) received by pid 3760

Origin: (TCP) 10.0.0.1:32155-192.168.0.1:13419

192.168.0.1:1135->172.16.0.2:12865 (17) sent by pid 3760

Origin: (TCP) 10.0.0.1:32155-192.168.0.1:13419

$192.168 .0 .1: 1135->172 \cdot 16 \cdot 0 \cdot 2: 59850$ (17) sent by pid 3760

Origin: (TCP) 10.0.0.1:32155-192.168.0.1:13419

192.168.0.1:1135->172.16.0.2:10435 (17) sent by pid 3760

Origin: (TCP) 10.0.0.1:32155-192.168.0.1:13419

192.168.0.1:1135->172.16.0.2:4577 (17) sent by pid 3760

Origin: (TCP) 10.0.0.1:32155-192.168.0.1:13419

Figure 11: Logged network traffic of process 3760 on bliss: control traffic with the master and DoS traffic to the victim

\section{Related Work}

\subsection{Packet Source Determination}

In normal operation, a host receiving packets can determine their source by direct examination of the source address field in the IP packet header. Unfortunately, this address is easy to falsify, making it simple for attackers to send packets that have their source effectively hidden. This is more common for one-way communication, such as the UDP and ICMP packets used in denial-ofservice attacks, but has been of use in attacks using TCP streams in which the TCP sequence numbers are guessable $[22,2]$. There has been significant recent research in how to locate the source of such packets, primarily motivated by distributed denial-of-service (DDoS) attacks in early February of 2000. While it is generally recommended that routers be configured to perform ingress or egress routing [13], it is clear from continuing denial-of-service attacks [21] that this is not widely done. There have been other methods proposed to perform filtering to limit the effect of such attacks [25, 15, 41].

As it is currently not possible to prevent such attacks, recent work has focused on how to locate the source of attacks. Some methods add or collect information at routers to allow traceback of DoS traffic [30, 38, 8, 33]. Other methods add markings to the packets to probabilistically allow determination of the source given sufficient packets $[31,34,24,9,10,1]$, or forward copies of packets, encapsulated in ICMP control messages, directly to the destination [40]. A more innovative method uses counter-DoS attacks to locate the source of ongoing attacks [5]. While we do not require that these schemes be available, we can make effective use of the traceback information they provide in the event spoofed packets are used.

\subsection{Correlating Streams}

Up to now, research addressing determination of the source of a connection chain has mainly focused on correlating streams of TCP connections observed at dif- 
ferent points in the network. The initial work in matching streams constructed thumbprints of each stream based on content [35]. While this technique could effectively match streams, it would be ineffective in compressed or encrypted streams as are common today. Other work compared the rate of sequence number increase in TCP streams as a matching mechanism, which can work as long as the data is not compressed at different hops and does not see excessive network delay [42]. Another technique, which relies solely on the timing of packets in a stream, is effective against encrypted or compressed streams of interactive user data [44]. This work was originally intended for intrusion detection purposes but was also proposed as an effective method for finding the source of connection chains. While performing stream matching might be effective in some cases, such methods rely on examining network information, and might be vulnerable to the same methods that can be used to defeat network intrusion detection systems [27]. More recent work has examined the effectiveness of attackers attempting to defeat stream matching by adding delay or additional packets to the data stream, but did not propose a method of directly matching streams [11].

\section{Limitations and Future Work}

This paper presents a first attempt at a mechanism designed to address the problem of determining host causality. While it is progress in a forward direction, it is not a complete solution to a problem, though its use could prove beneficial in many cases. We hope that discussion of the limitations will foster other research on the problem.

While available origin information is maintained for processes that utilize setlogin, there are other mechanisms that attackers can use to start processes on a system. Remotely, attackers might gain access to a system using processes that service network requests, such as mail, web, or ftp servers. Exploits such as buffer overflows against these processes can produce user shells for the attacker, bypassing the system call. In these cases, origin information will not be properly recorded. For these cases the question arises when exactly to set the origin information so that it is meaningful. Furthermore, an attacker who gains access to a system might use a cron or at job to create a process after the attacker has logged off; this would also result in processes that lack the correct origin information. A solution to this problem might be to include origin information in the file system so that when the new process was started the appropriate location information was available.

Sometimes login servers can open a second connection to the client for outof-band data. Currently this scenario is not handled in the design. However, its seems that in the worst case the wrong port is recorded for the origin within the modified accept system call.

An attacker also might use a covert channel between processes to obscure the proper location information. In this scenario, an attacker, who perhaps enters the system though a mechanism that invokes setlogin and whose processes 
therefore have correct origin information, uses some form of IPC to cause a process that has other origin information to send data into the network. This is a difficult problem to deal with, as it has always been [14], and we do not have an immediate solution for it. Any process that listens on a covert channel needs to have been started either locally or remotely, however, and in the case of an external attacker, most likely remotely. Thus, any outgoing traffic from that process will still be logged.

When two processes with different remote origins communicate with each other, perhaps using IPC or a shared file or shared memory, and data is later sent from either process to the network, we would like to be able to determine which of the processes was responsible for the output or whether both of them were the cause. To do this, the kernel would have to be able to trace the information flow of each process or more specifically the program the process is running. In the general case, this is an undecidable problem. One solution would be to only allow "origin information-friendly" applications to be run on a system, where the information flow has been determined in advance through static analysis. Apart from a large up-front cost in the static analysis, strict and reliable access control mechanisms are needed to enforce this approach. A second solution lies in allowing more than one possible origin for a process and devising a model that governs propagation of those origins based on information exchanges between processes and space limitations. This second solution is part of our current research and outside the scope of this paper.

While our implementation only operates on TCP and UDP packets, any protocol could be used by an attacker. For example, some DDoS tools use ICMP messages to send control messages over the network. In this case, an attacker would either have to modify the routines for ICMP processing in the kernel or may have to sniff the incoming traffic using a library like libpcap. If the attacker has modified the kernel to listen to and process these messages, there seems to be little that can be done to establish the origin information for a process, because if the kernel can been modified by the attacker, the origin information can be tampered with as well. In the latter case one can check for open BPF filters and also be aware of processes that utilize other protocols or do not receive network packets from the networking stack but rather through the packet filter.

The mechanism for keeping track of the inheritance line for a process needs to be improved. The current mechanism, while very simple to implement, is the only part of the modifications we made that affects the system in a noticeable fashion. One problem is that with each new child process, more information needs to be stored, even though it is small. Once a separate data structure for keeping inheritance lines is used, a simple improvement would be to delete inheritance lines or parts of it where all the processes involved have terminated. However, overall management of the inheritance lines remains as future work.

In the event of a system compromise, in which an attacker gains root capabilities, the origin information in the kernel and recorded information in the file system is just as vulnerable to modification or deletion as any other kernel or file system information. We consider this outside of the scope of our work, but 
point to other work that attempts to make audit information survive such attacks [32], and suggest that current forensics tools could be modified to recover the altered origin information in some cases.

Finally, as mentioned above, the packet logging system is a prototype only; a more effective design would be to include the logging mechanism in the kernel itself. Instead of sniffing for outgoing packets, writes to a network socket would cause the outgoing packet to be logged before the socket could be closed, alleviating the problem with trying to find the source of UDP packets mentioned in Section 8 above. Additionally, the current mechanism logs all TCP SYN and UDP packets, creating a denial-of-service opportunity for attackers to fill up disk space, so a more selective approach to recording packets is clearly in order, where possible.

\subsection{Future work in Host Causality}

Even though the origin information was designed with network traceback in mind, there are other applications or foundations for new modifications of the system:

- A system administrator can use the origin information to determine the origins of all running processes and identify ones that have a very unusual source. This can lead to the discovery of running DDoS clients on a machine, for example.

- The origin information can be incorporated into the file system. By storing a process's origin information with a file whenever the process writes to the file system. Not only can this help in solving the problem with cron and startup scripts, but it can also aid in locating suspicious programs in users' home directories. This would be especially effective with logging file systems, so that the changes in files could be tracked by location as well.

- Origin information adds another dimension to access control. Access control mechanisms can be altered so that they take origin information into account and grant certain privileges only when certain origin conditions are met.

- Statistics based on origin of processes can be gathered, which can be used to profile normal system behavior or to locate trends that may help in better system administration.

Origin information may well benefit in other security related fields. The prospect of access control in combination with origin information seems to be an especially interesting area. Research in that direction may well improve overall robustness of the origin mechanism itself. 


\section{Conclusion}

In this paper, we have introduced the notion of host causality as a mechanism to complement current research in network traceback. With the addition of origin information to a process, we have developed a mechanism that, with only minor changes to the given system adds audit data allowing traceback of information through a host. The two examples show that important information for network traceback can be obtained with origin information and the new logging possibilities that result from that.

The work presented here is only the start of work in the overall area. We have identified many limitations of our mechanism, and outlined what future work needs to be done to better address the problem. Host causality is not a complete solution to all the problems that occur in tracing connections through a network, but providing solutions could prove a valuable tool to help improve security in a future networking environment.

\section{Acknowledgements}

We are grateful for the feedback of the anonymous reviewers in helping the development of this paper. 


\section{References}

[1] M. Adler. Tradeoffs in Probabilistic Packet Marking for IP Traceback. In Proceedings of the 34th ACM Symposium on Theory of Computing (STOC), 2002.

[2] S. M. Bellovin. Security Problems in the TCP-IP Protocol Suite. Computer Communications Review, 19(2):32-48, April 1989.

[3] F. Buchholz, T. Daniels, B. Kuperman, and C. Shields. Packet Tracker Final Report. Technical Report 2000-23, CERIAS, Purdue University, 2000.

[4] F. Buchholz and C. Shields. Providing Process Origin Information to Aid in Network Traceback. In Proceedings of the 2002 USENIX Annual Technical Conference, 2002.

[5] H. Burch and B. Cheswick. Tracing Anonymous Packets to their Approximate Source. In Proceedings of the 14th Conference on Systems Administration (LISA-2000), New Orleans, LA, December 2000.

[6] B. Carrier and C. Shields. A Recursive Session Token Protocol for use in Computer Forensics and TCP Traceback. In Proceedings of the IEEE Infocomm 2002, June 2002. To appear.

[7] CERT Coordination Center. CERT/CC Statistics 1988-2003. http://www . cert.org/stats/cert_stats.html, 2003.

[8] Characterizing and Tracing Packet Floods Using Cisco Routers. http: //www.cisco.com/warp/public/707/22.html.

[9] D. Dean, M. Franklin, and A. Stubblefield. An algebraic approach to ip traceback. "ACM Transactions on Information and System Security (TISSEC)", 5(2):119-137, May 2002.

[10] T. W. Doeppner, P. N. Klein, and A. Koyfman. Using Router Stamping to Identify the Source of IP Packets. In Proceedings of the rth ACM Conference on Computer and Communications Security, pages 184-189, Athens, Greece, November 2000.

[11] D. Donoho, A. G. Flesia, U. Shankar, V. Paxson, J. Coit, and S. Staniford. Multiscale stepping-stone detection: Detecting pairs of jittered interactive streams by exploiting maximum tolerable delay. In Proceedings of the 2002 Recent Advances in Intrusion Detection (RAID), 2002.

[12] D. Farmer and W. Venema. The Coroner's Toolkit (TCT). http://www . fish.com/tct/.

[13] P. Ferguson and D. Senie. Network Ingress Filtering: Defeating Denial of Service Attacks which employ IP Source Address Spoofing. Technical Report RFC 2827, Internet Society, May 2000. 
[14] V. Gligor. A Guide to Understanding Covert Channel Analysis of Trusted Systems. Technical Report NCSC-TG-030, National Computer Security Center, Ft. George G. Meade, Maryland, U.S.A., November 1993. Approved for public release: distribution unlimited.

[15] J. Ioannidis and S. M. Bellovin. Pushback: Router-Based Defense Against DDoS Attacks. In Proceedings of the 2002 Network and Distributed System Security Symposium, San Diego, CA, February 2002.

[16] H. T. Jung, H. L. Kim, Y. M. Seo, G. Choe, S. L. Min, C. S. Kim, and K. Koh. Caller Identification System in the Internet Environment. In Proceedings of the UNIX Security Symposium IV, pages 69-78, 1993.

[17] S. C. Lee and C. Shields. Tracing the Source of Network Attack: A Technical, Legal, and Societal Problem. In Proceedings of the 2001 IEEE Workshop on Information Assurance and Security, West Point, NY, June 2001.

[18] B.N. Levine and C. Shields. Hordes - A Multicast Based Protocol for Anonymity. Journal of Computer Security, 10(3):213-240, 2002.

[19] M.K. McKusick, K. Bostic, M.J. Karels, and J.S. Quarterman. The Design and Implementation of the 4.4 BSD Operating System. Addison Wesley, Boston, MA, 1996.

[20] D.L. Mills. Network Time Protocol. RFC 1059, July 1988.

[21] D. Moore, G. Voelker, and S. Savage. Inferring Internet Denial of Service Activity. In Proceedings of the 2001 USENIX Security Symposium, Washington D.C., August 2001.

[22] R.T. Morris. A Weakness in the 4.2BSD Unix TCP-IP Software. Technical Report 17, AT\&T Bell Laboratories, 1985. Computing Science Technical Report.

[23] Distributed Attack Tools Section. http://packetstorm.securify.com/ distributed/.

[24] K. Park and H. Lee. On the Effectiveness of Probabilistic Packet Marking for IP Traceback Under Denial of Service Attack. In Proceedings of the IEEE INFOCOM 2001, pages 338-347, April 2001.

[25] K. Park and H. Lee. On the Effectiveness of Route-Based Packet Filtering for Distributed DoS Attack Prevention in Power-Law Internets. In Proceedings of the 2001 ACM SIGCOMM, San Diego, CA, August 2001.

[26] J. Picciotto. The Design of An Effective Auditing Subsystem. In Proceedings of the IEEE Symposium on Security and Privacy, pages 13-22, 1987. 
[27] T. Ptacek and T. Newsham. Insertion, Evasion, and Denial of Service: Eluding Network Intrusion Detection. Technical report, Secure Networks, Inc., January 1998.

[28] M. Reed, P. Syverson, and D. Goldschlag. Anonymous Connections and Onion Routing. IEEE Journal on Selected Areas in Communication Special Issue on Copyright and Privacy Protection, 1998.

[29] M. K. Reiter and A. D. Rubin. Crowds: Anonymity for Web Transactions. ACM Transactions on Information and System Security, 1(1):66-92, November 1998.

[30] J. Rowe. Intrusion Detection and Isolation Protocol: Automated Response to Attacks. Presentation at Recent Advances in Intrusion Detection (RAID), 1999.

[31] S. Savage, D. Wetherall, A. Karlin, and T. Anderson. Practical Network Support for IP Traceback. In Proceedings of the 2000 ACM SIGCOMM Conference, August 2000.

[32] B. Schneier and J. Kelsey. Secure Audit Logs to Support Computer Forensics. ACM Transactions on Information and System Security, 1(3), 1999.

[33] A. C. Snoeren, C. Partridge, L. A. Sanchez, C. E. Jones, F. Tchakountio, and W. T. Strayer S. T. Kent. Hash-Based IP Traceback. In Proceedings of the 2001 ACM SIGCOMM, San Diego, CA, August 2001.

[34] D. X. Song and A. Perrig. Advanced and Authenticated Marking Schemes for IP Traceback. In Proceedings of the IEEE Infocomm 2001, April 2001.

[35] S. Staniford-Chen and L.T. Heberlein. Holding Intruders Accountable on the Internet. In Proceedings of the 1995 IEEE Symposium on Security and Privacy, pages 39-49, Oakland, CA, May 1995.

[36] W. R. Stevens. Unix Network Programming, volume 1. Prentice Hall PTR, second edition, 1998.

[37] W.R. Stevens. Advanced Programming in the UNIX Environment. AddisonWesley, Reading, MA, 1993.

[38] R. Stone. CenterTrack: An IP Overlay Network for Tracking DoS Floods. In Proceedings of the 9th USENIX Security Symposium, Denver, CO, August 2000 .

[39] G.R. Wright and W.R. Stevens. TCP/IP Illustrated Volume 2, The Implementation. Addison Wesley, Boston, MA, 1995.

[40] S. F. Wu, L. Zhang, D. Massey, and A. Mankin. Intention-Driven ICMP Trace-Back. IETF Internet draft, February 2001. Work in progress. 
[41] A. Yaar, A. Perrig, and D. Song. Pi: A Path Identification Mechanism to Defend against DDoS Attacks. In Proceedings of the IEEE Symposium on Security and Privacy, May 2003.

[42] K. Yoda and H. Etoh. Finding a Connection Chain for Tracing Intruders. In Proceedings of the 6th European Symposium on Research in Computer Security (ESORICS 2000), October 2000.

[43] ZDNet Special Report: It's War! Web Under Attack. http://www.zdnet. com/zdnn/special/doswebattack.html, February 2000.

[44] Y. Zhang and V. Paxson. Detecting Stepping Stones. In Proceedings of the 9th USENIX Security Symposium, Denver, CO, August 2000. 